\title{
Increased expression of IL-33 is found in the lower airways of patients with seasonal allergic rhinitis and is not related to natural allergen exposure.
}

Amaryllis Haccuria ${ }^{1}$, Alain Van Muylem ${ }^{1}$, Andrei Malinovschi ${ }^{2}$, Joanne rasschaert ${ }^{3}$, myrna virreira $^{3}$, Nicolas Bruffaerts ${ }^{4}$, Marijke Hendrickx ${ }^{4}$, and Alain Michils ${ }^{1}$

${ }^{1}$ CUB Erasme, Université Libre de Bruxelles

${ }^{2}$ Uppsala University

${ }^{3}$ Universite Libre de Bruxelles Faculte de Medecine

${ }^{4}$ Sciensano

June 9, 2020

\begin{abstract}
Introduction The IL-33 pathway involved in the development of type-2 airway inflammation is activated in allergic asthma patients. According to the "one airway, one disease" concept, the IL-33 pathway should also be activated in the airways of allergic rhinitis patients. Material and methods We compared the levels of IL-33 and its mRNA precursor, in induced sputum of patients suffering from seasonal allergic rhinitis $(n=27)$ with those measured in patients with seasonal allergic asthma $(n=23)$, and in healthy controls $(\mathrm{n}=17)$, in and out of the pollen season. Results IL-33 levels were higher in sputum supernatants of allergic rhinitis (median 9.4 pg.ml-1, range $0-51.1$ pg.ml-1) and asthma patients (5.2 pg.ml-1, range $0-45.4$ pg.ml-1) when compared to controls (median 0 pg.ml-1, range $0-13$ pg.ml-1; p $<0.001$ ), and the same was observed with qPCR for IL-33. IL-33 levels were similar in patients suffering from allergic rhinitis (median 4.9 pg.ml-1 vs 7.1 pg.ml- $1, \mathrm{p}=0.256$ ) or allergic asthma patients (median 3.6 pg.ml-1 vs 3.2 pg.ml-1, p=0.61) in and out of pollen seasons. Conclusion IL-33 is detectable in the lower airways of allergic rhinitis patients, to similar levels than in asthma patients, and appears to be independent of natural variation in allergen exposure. Those findings could reflect a silent epithelial dysfunction in the lower airways of allergic rhinitis patients without asthma, further confirming the «one airway, one disease» theory linking asthma and rhinitis.
\end{abstract}

\section{Hosted file}

HA-ALLERGY-DEFINITIF.docx available at https://authorea.com/users/331645/articles/458254increased-expression-of-il-33-is-found-in-the-lower-airways-of-patients-with-seasonalallergic-rhinitis-and-is-not-related-to-natural-allergen-exposure 